\title{
A Method for Stable Indoor Positioning using Beacons
}

Young Hyun Kim* and Suk-Ho Lee

\author{
Department of Software Engineering, Dongseo University, Busan 617 - 716, Korea; petrasuk@gmail.com
}

\begin{abstract}
Indoor navigation finds its usage in indoor shopping, conferences, or evacuation in case of emergency case. The fundamental technology for indoor navigation is indoor positioning. The main problem in indoor positioning is how to calculate an accurate position from the wireless signals emitted by indoor access points. Since the GPS cannot be used in indoor environments, other devices that can be used as access points have to be utilized. One of those devices, which have gained a lot of popularity, recently, due to its low battery consumption and wide signal range is the Beacon. However, the Beacon contains also much error in the estimation of the distance. Therefore, if used with the conventional trigonometric method to estimate the position, the position will contain much error. Therefore, in this paper, we propose a stable indoor positioning method using multiple sets of Beacons. The proposed method alleviates the position error by a signal processing technique. Experimental results verify the validness of the proposed method.
\end{abstract}

Keywords: Beacons, Indoor Positioning, Signal Processing

\section{Introduction}

The self-positioning technique lies in the heart of navigation. For example, the self-positioning using Global Positioning System (GPS) makes the navigation using smartphone apps possible. In Korea, several navigation apps such as the T-Map made by SK Planet have already many users. Furthermore, there is an increasing demand for indoor positioning using personal devices such as the smart phone. For example, Google is trying to launch a new indoor positioning service similar to the current outdoor positioning in Figure 1.

Another important application of indoor positioning is the guide to an exit in case of emergency. It is known that people cannot find easily the exit in case of fire, because of the confusion and the invisibility caused by the smoke. For example, many people died in the fire accident of the Goyang complex terminal as they could not find the exit due to invisibility. Since GPS information cannot be used in indoor positioning, the position under consideration has to be calculated from the distances of several access points which send wireless signals to the receiver.

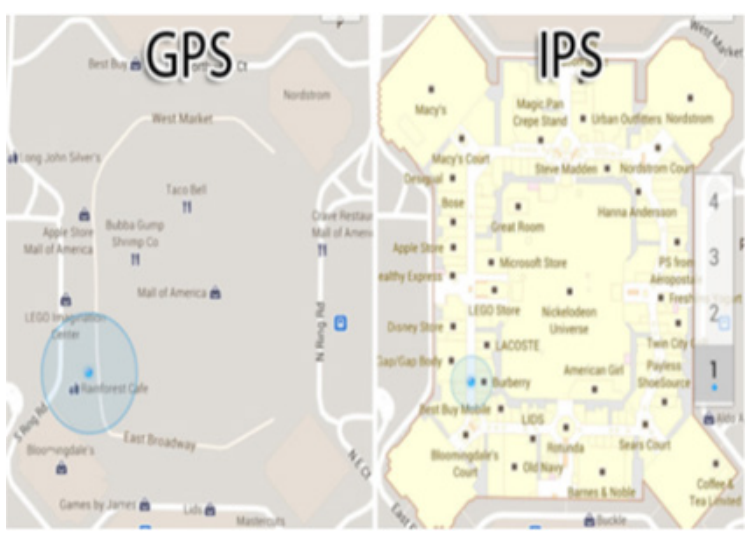

Figure 1. Outdoor and Indoor positioning service by Google.

One of the popular devices that can be used as an access point is the Beacon. Recently, the Beacon has gained much attention due to its low consumption of the battery and its wide signal range. However, since there exists error in the measurement of the distance estimated from the signal sent from the Beacon, the measured position also contains errors. To obtain stable position information, the error has

\footnotetext{
*Author for correspondence
} 
to be reduced by signal processing techniques. Therefore, in this paper, we propose a method which alleviates the position error by using a multiple set of Beacons. Instead of the trigonometric measurement, we calculate the intersection of two circles made by estimation for position estimation. Due to the use of multiple sets of Beacons, multiple estimations of the position are performed. After that, a constrained weighted averaging method is applied on the estimated positions to obtain a single reliable estimate. The weighting function is designed such that the large outliers are excluded from the estimation and that the errors in the multiple estimates are cancelled by each other. Experimental results show that the use of multiple Beacons results in a more accurate estimation of the position.

\section{Problems in the Positioning using Beacons}

Beacons are devices which work using the Bluetooth Low Energy (BLE) technology. Since most smart phones support the Bluetooth 4.0 technology, the signal emitted by the Beacons can be received by the smart phone, and the distance can be estimated from the strength of the signal. Using three Beacons, the position of the smart phone relative to the beacons can be estimated by the trigonometric measurement. Conventional methods for estimating the position from the signals sent by three different signal stations use the trigonometric measurement ${ }^{1-3}$. In these approaches, the distances from the signal stations to the receiver are calculated first. Then, the position of the receiver is at the intersection of the three circles centered at the stations with radiuses equal to the measured distances. Normally, if the distances are measured accurately, then the receiver will be at the intersection of the three circles. This situation is shown in Figure 2.

However, since there exists error in the measurement, the position lies not at the intersection, but normally inside a region made by the made by the intersections of every two circles. Figure 3 shows this situation. Here, the intersections of every two circles are denoted as A, B and $\mathrm{C}$. Then, a circle can be drawn which goes through all the intersection points. The position of the receiver is calculated to be at the center of this circle.

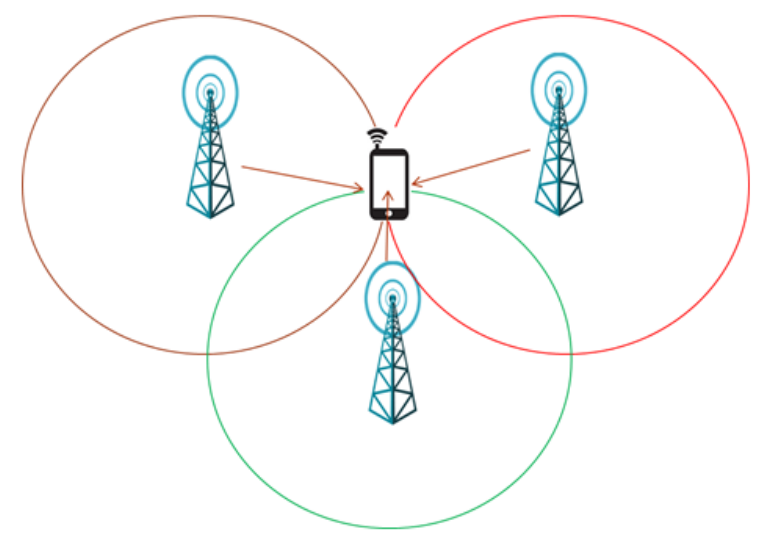

Figure 2. Showing the concept of the trigonometric estimation using three signal stations.

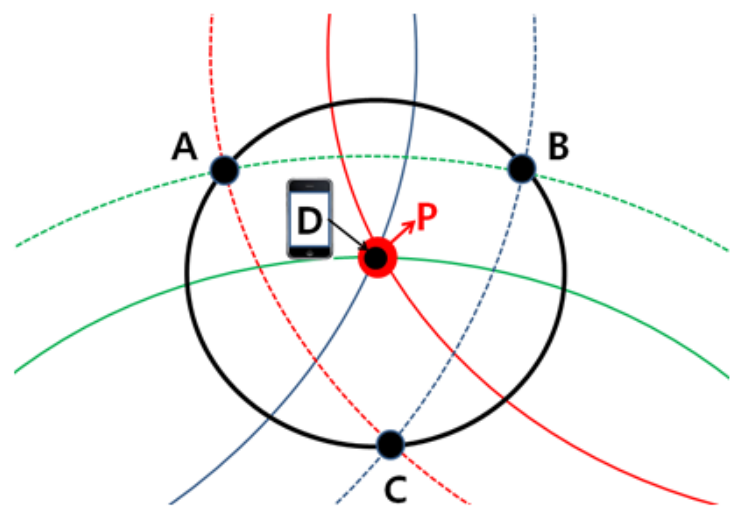

Figure 3. Showing the concept of the trigonometric estimation with distance measurements containing errors.

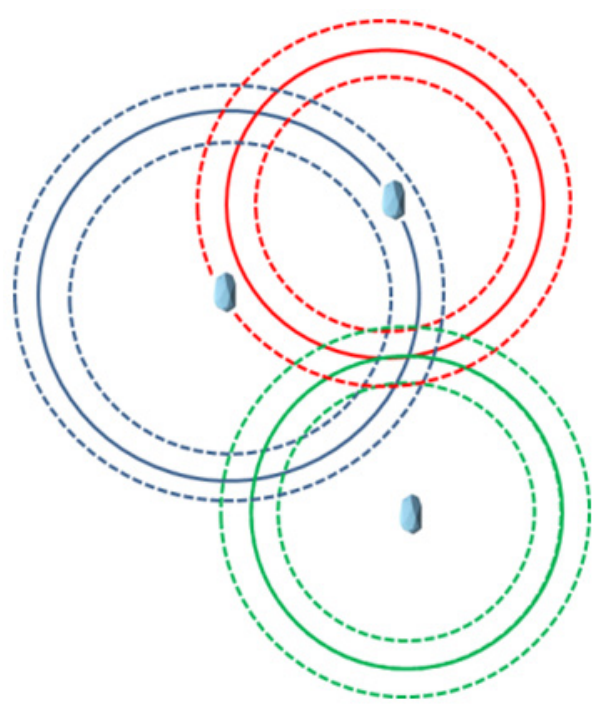

Figure 4. Beacons with positions inclined in one direction. 
However, the problem with Beacons is that the error in the measured distances is rather large. It is known that the deviation is $5-6 \mathrm{~cm}$ for a distance of $20 \mathrm{~cm}$. This error increases as the distance gets larger. It becomes $15 \mathrm{~cm}$ for a distance of 1 meter, and 2-3 meters for a distance of 10 meters. Due to the incorrect distance measurement of the Beacon device, the position estimated by the trigonometric method also becomes incorrect. Furthermore, if the relative positions of the Beacons used in the measurement are inclined in one direction as shown in Figure 4, then, the true position of the smartphone can be outside of the circle that goes through the intersection points of the circles with radiuses obtained by the estimated ranges of the Beacons as shown in Figure 5. Therefore, we propose a signal processing method using more than three Beacons to get a more precise position.

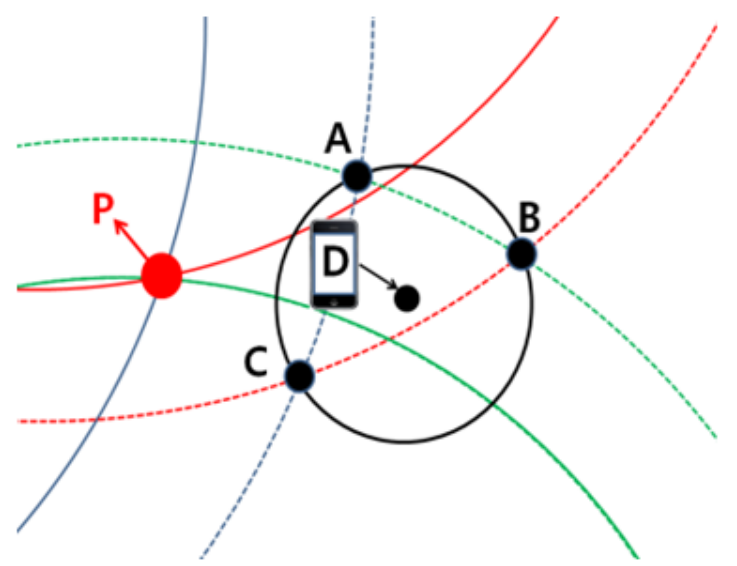

Figure 5. Comparing the position of the true position $(\mathrm{P})$ with the estimated position (D) using Beacons inclined in one direction.

\section{Proposed Method}

The proposed method uses a constrained weighted averaging method instead of the trigonometric measurement for position estimation. It uses multiple of Beacons (more than 3 Beacons) to obtain a single position. The weighting function used in the weighted averaging is designed such that the large outliers are excluded from the estimation and that the errors in the multiple estimates are cancelled by each other.

\subsection{Weighted Averaging based Position Estimation}

To compute the center point from three stations, first, the intersections of every two circles are computed. Figure 6 shows how two circles are intersected at two points. Here, $r_{1}$ and $r_{2}$ denote the radiuses of the two circles, and $d$ denotes the distance between the centers of the two circles. The coordinates of the two intersection points are denoted as $\left(a_{1}, b_{1}\right)$ and $\left(a_{2}, b_{2}\right)$, and can be calculated by the following equations:

$a_{1}=\frac{l}{d}\left(x_{2}-x_{1}\right)+\frac{h}{d}\left(y_{2}-y_{1}\right)+x_{1}$

$b_{1}=\frac{l}{d}\left(y_{2}-y_{1}\right)-\frac{h}{d}\left(x_{2}-x_{1}\right)+y_{1}$

and

$a_{2}=\frac{l}{d}\left(x_{2}-x_{1}\right)-\frac{h}{d}\left(y_{2}-y_{1}\right)+x_{1}$

$b_{2}=\frac{l}{d}\left(y_{2}-y_{1}\right)+\frac{h}{d}\left(x_{2}-x_{1}\right)+y_{1}$

where, $d, l$ and $h$ are defined as follows.

$$
\begin{aligned}
& d=\sqrt{\left(x_{2}-x_{1}\right)^{2}+\left(y_{2}-y_{1}\right)^{2}} \\
& l=\frac{r_{1}^{2}+r_{2}^{2}+d^{2}}{2 d} \\
& h=\sqrt{r_{1}^{2}-l^{2}}
\end{aligned}
$$

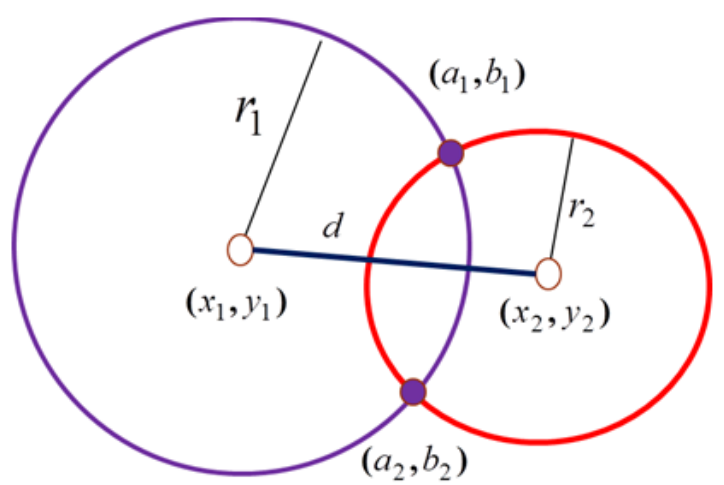

Figure 6. Showing two intersection points of two circles.

By computing all the intersection points of every two circles, we get six points $\left(\mathbf{z}_{1} \sim \mathbf{z}_{6}\right)$ as shown in Figure 
7. Then, the mean point $(\boldsymbol{c})$ of all the intersection points can be obtained. However, this point too can be deviated from the real center position. To correct the center point, we calculate the $L_{2}$ norm distance from the mean point to all the intersection points. Then, we eliminate the intersection points that lie far from the center point.

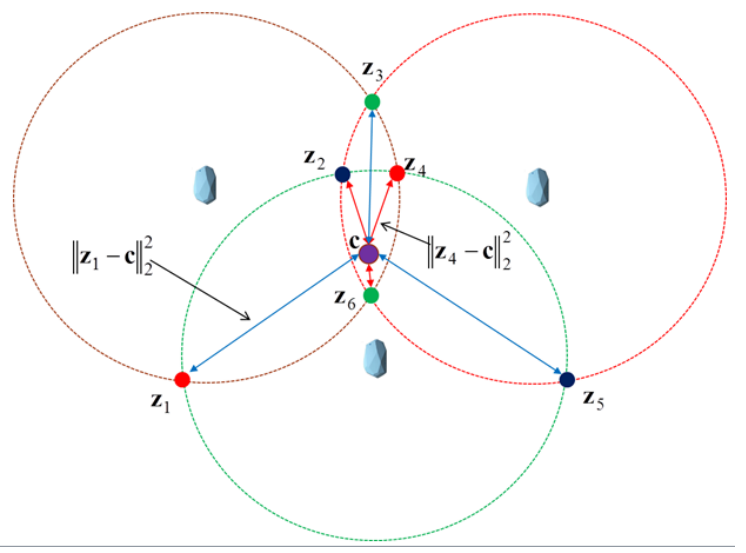

Figure 7. Estimating the position using 3 Beacons.

We do this by choosing the subset consisting of 3 elements, for which the sum of the $L_{2}$ norms is the smallest:

$\hat{s}=\arg \min _{s \subset S} \sum_{\mathbf{z}_{k} \in s}\left\|\mathbf{z}_{k}-\mathbf{c}\right\|_{2}^{2}$

where

$S=\left\{\mathbf{z}_{1}, \mathbf{z}_{2}, \mathbf{z}_{3}, \mathbf{z}_{4}, \mathbf{z}_{5}, \mathbf{z}_{6}\right\}$

and $s$ denotes any subset of $S$ containing 3 elements. Then, the corrected center point is calculated as, $\hat{\mathbf{c}}=\frac{1}{3} \sum_{\mathbf{z}_{k} \in s} \mathbf{z}_{k}$

where the recalculation has been done using only the points that are in the subset $s$.

If we have three Beacons, we obtain one center point by the previously mentioned method. In the case that we have 4 Beacons we get ${ }_{4} C_{3}=4$ estimated center points. Using 6 Beacons, the number becomes ${ }_{6} C_{3}=20$ points in Figure 8. This shows the fact that the number of the estimated center points increases geometrically as more Beacons are involved in the calculation.

Using these 20 center points, we can compute the weighted average of those points to get the final position.

We do this by the following minimization:

$\hat{\mathbf{c}}=\arg \min _{\mathbf{c}} \sum_{k=1}^{N}\left(\mathbf{c}-w_{k} \mathbf{c}_{k}\right)^{2}$
Here, $\boldsymbol{c}_{k}$ denotes the estimated position by every set of three Beacons, $N$ denotes the number of sets, $w_{k}$ denotes a possible confidence weight value, and $\hat{\boldsymbol{c}}$ is the final estimated position. The weight $w_{k}$ is given a large value if the corresponding estimated $\boldsymbol{c}_{k}$ value is near the previously computed weighted mean and small if it is far from it. We use a two-step algorithm to compute the final center point. First, we let all the weighting values 1 . Then, we compute the center point using these weighting values. Next, we update the weighting function. These two steps are iterated for a predefined number of iteration. Thus, the algorithm can be described as:

- Initial: Let the initial weighting values be $w_{k}=1$ for all $k$.

- Step 1: Estimate the position:

$\hat{\mathbf{c}}=\arg \min _{\mathbf{c}} \sum_{k=1}^{N}\left(\mathbf{c}-w_{k} \mathbf{c}_{k}\right)^{2}$

- Step 2: Compute the weighting function:

$$
w_{k}=\left\{\begin{array}{cl}
\frac{\frac{1}{N} \sum_{m}\left\|\mathbf{c}-\mathbf{c}_{m}\right\|_{2}}{\left\|\mathbf{c}-\mathbf{c}_{k}\right\|_{2}} & \text { if }\left\|\mathbf{c}-\mathbf{c}_{k}\right\|_{2} \leq \frac{5}{N} \sum_{m}\left\|\mathbf{c}-\mathbf{c}_{m}\right\|_{2} \\
0 & \text { if }\left\|\mathbf{c}-\mathbf{c}_{k}\right\|_{2}>\frac{5}{N} \sum_{m}\left\|\mathbf{c}-\mathbf{c}_{m}\right\|_{2}
\end{array}\right.
$$

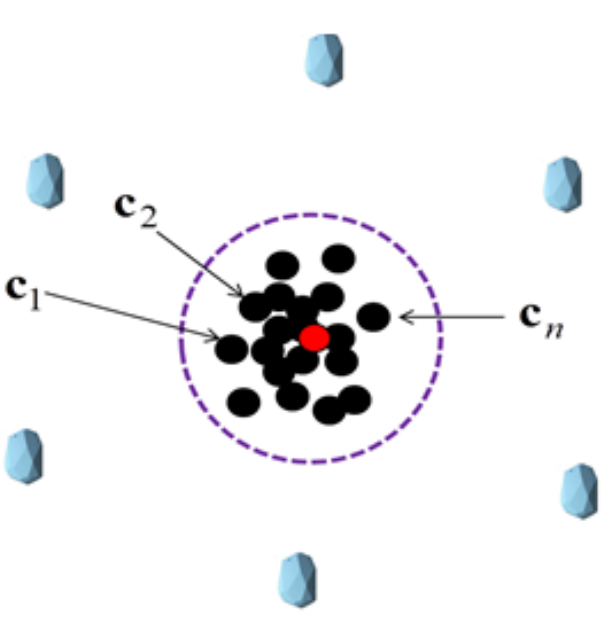

Figure 8. Estimating the position using 6 Beacons.

where, $\boldsymbol{c}$ denotes the mean calculated in the previous step, $\boldsymbol{c}_{m}$ denotes the estimated position of all Beacons, and $\boldsymbol{c}_{k}$ is the estimated position of the Beacon under consideration for which the weighting value $w_{k}$ is calculated. The weighting value is large if the corresponding position measured by the Beacon is near the previously computed main center point. It becomes zero, if the measured position is too far from the previously computed main center point. 
- Repeat Step 1 and Step 2 for a predefined number of iteration.

The finally measured position $\hat{\boldsymbol{c}}$ can be also seen as a denoised version of all the measured $\boldsymbol{c}_{k}$ values.

\subsection{Denoising Property of Proposed Method}

Here, we explain why the proposed method obtains a more accurate position using the well-known denoising property. Suppose, we have $m^{2}$ estimates $\left(\boldsymbol{c}_{k}\right)$ of the real position $\left(\boldsymbol{s}_{k}\right)$, each containing error components $\boldsymbol{n}_{k}$.

$\mathbf{c}_{k}=\mathbf{s}_{k}+\mathbf{n}_{k}$

Further, suppose that the errors contained in each estimates have a Gaussian distribution with variation of $\sigma^{2}$. Let, the set of all estimates be $A=\left\{\boldsymbol{c}_{1}, \boldsymbol{c}_{2}, \boldsymbol{c}_{3}, \ldots \boldsymbol{c}_{N}\right\}$.

Then, it is well-known that the variation of the error in $A$ becomes:

$$
\operatorname{var}(A)=E\left[(A-E(A))^{2}\right]=\frac{\sigma^{2}}{m}
$$

where

$E(A)=\frac{1}{m^{2}} \sum_{k=1}^{m^{2}} \mathbf{s}_{k}$

This shows that the variation in the error is reduced from $\sigma^{2}$ to $\sigma^{2} / m$. Of course, it is not verified that the error in the Beacon has a Gaussian distribution.

\section{Experimental Results}

We experimented with 4 Beacons for the proposed method. We compared the results with the trigonometric measurement using 3 Beacons. The error comparison is summarized in Table 1. For example for a distance of 2, the trigonometric estimation shows an average error of 1.5 meter, while our algorithm reduces it to 0.5 meter. Using more than 4 Beacons, the error can be further reduced.

Table 1. Comparison of the Average error in the estimated position (Unit: Meter).

\begin{tabular}{lcc}
\hline $\begin{array}{c}\text { Distance of } \\
\text { the Beacon }\end{array}$ & $\begin{array}{c}\text { Trignometric estimation } \\
\text { with 3 Beacons }\end{array}$ & $\begin{array}{c}\text { Proposed } \\
\text { method }\end{array}$ \\
\hline 2 & 1.5 & 0.5 \\
3 & 1.9 & 0.6 \\
5 & 3.2 & 0.9 \\
\hline
\end{tabular}

\section{Acknowledgement}

This research was supported by the University for Creative Korea-1 Project (CK-1) and the Leaders in IndustryUniversity Cooperation (LINC) Project through the Ministry of Education.

\section{References}

1. $\mathrm{Gu}$ Y, Lo A, Niemegeers I. A survey of indoor positioning systems for wireless personal networks. IEEE Communications Surveys Tutorials. 2009; 11(1):13-32.

2. Anastasi G, Bandelloni R, Conti M, Delmastro F, Gregori E, Mainetto G. Experimenting an indoor bluetooth-based positioning service. Proceedings of 23rd International Conference on Distributed Computing Systems; RI; Russia; IEEE: 2003 p. 480-3.

3. Chawathe SS. Beacon placement for indoor localization using Bluetooth. 11th International IEEE Conference on Intelligent Transportation Systems (ITSC); Beijing; China; IEEE: 2008. p. 980-5. 